\title{
Influence of magnetic field and working voltage on the tripping performance in spherical cylindrical ECP
}

\author{
Zhang Jianping ${ }^{1,2,{ }^{*}}$ Ren Baodong ${ }^{2}$ \\ ${ }^{1}$ College of Energy and Mechanical Engineering, Shanghai University of Electric Power, Shanghai 200090, China \\ ${ }^{2}$ School of Mechanical Engineering, University of Shanghai for Science and Technology, Shanghai 200093, China
}

\begin{abstract}
In order to further improve the trapping effect of fine particles, a new electrostatic cyclone precipitator (ECP) with magnetic confinement was proposed, the overall efficiencies of fine particles under different operating conditions were numerically simulated, and the influence of working voltage on the dustremoval effect of fine particles with and without magnetic field were discussed. The results show that increasing working voltage or magnetic induction intensity improves the trapping performance of spherical cylindrical magnetically confinement ECP, and the lifting effect gradually weakens while increasing the same amplitude. The results can offer technical reference for the optimization design of greatly improving the ECP dust-removal performance.
\end{abstract}

\section{Introduction}

A large number of fossil fuels are consumed, and excessive dust-containing smoke is emitted into the air, resulting in increasingly serious air pollution, which has become an important problem to be solved in the world [13]. There are many kinds of atmospheric pollutants, including $\mathrm{SO}_{2}, \mathrm{NO}_{\mathrm{x}}$ and atmospheric particulate pollutants, among which particulate pollutants have become the primary pollutants affecting the quality of air environment $[4,5]$. Fine particles seriously affect the air quality and endanger human health, and the environmental pollution caused by them is attracting more and more attention from all walks of life [6]. As an effective gassolid separation equipment for dust-containing flue gas, cyclone dust collectors are constantly being optimized and developed [7, 8].

Since the first cyclone dust collector was put into production in 1885 , it has been more than 100 years of industrial application history [9-12]. Due to the limited understanding of its dust removal mechanism and process characteristics, the cyclone dust collector has a low initial dust-removal efficiency, and the minimum particle size that can be separated is more than $40-60 \mu \mathrm{m}$ [13-16]. In the following half century, many researchers began to study the dust removal mechanism and the internal flow law of the cyclone separators. Cortes [17] analyzed the internal flow field of the cyclone separator with a new method based on the model research, and demonstrated that unstable flow and secondary flow existed in the separator. Then, a series of basic theories with far-reaching influence, such as the rotating circle theory [18], screening theory [19] and boundary layer separation theory [20], were proposed.

On the basis of these important theories, more scholars have begun to combine electromagnetic dust-removal technology with cyclone dust collectors. In the design of dust-removal equipments, an electrostatic cyclone type household vacuum cleaner named 'Tatsumaki cyclone' [11] and a small three-stage mobile gas-solid separator with greatly optimized structure and size [21] were manufactured. In the aspect of numerical simulation, Huang [22] obtained the critical path line which reflects the threshold position of magnetic collection by simulating the particle velocity contour, indicating that the application of magnetized net filter can promote the collection of ferromagnetic dust or aerosol to some extent without any additional power or pressure resistance. Zhang [23] applied Fluent to simulate the dust removal efficiency of the wire-pipe ESP in a magnetic field environment, and found that the magnetic field has a more obvious effect under the conditions of high gas velocity and low working voltage.

To sum up, the traditional electrostatic cyclone precipitator (ECP) is widely used in industry and has good removal effect on large particles, but the trapping performance of fine particles remains to be improved. Therefore, a novel spherical cylindrical ECP was designed to optimize the dust-removal effect by introducing magnetic field, the electromagnetic dust-removal performance of fine particles was explored by numerical simulation and data analysis, and satisfactory results were obtained.

\footnotetext{
*Corresponding author: jpzhanglzu@163.com
} 


\section{Numerical model}

\subsection{Structural model and its parameters}

When studying the movement of dust particles in the spherical cylindrical ECP, the following reasonable assumptions need to be made in order to simplify the calculation: the existence of dust particles does not change the gas flow field; the dust particles do not affect each other; the dust particles are spheres with equivalent diameters. Only on this basis can the flow field be solved.

The model size of the spherical cylindrical cyclone in this work is selected from the experimental model used in literature [24], and the structure diagram is shown in Figure 1. In order to introduce the electric field into the dust removal area, a circular cross-sectional corona wire with a length of $\mathrm{L}=260 \mathrm{~mm}$ is vertically added at the center position of the spherical cylindrical cyclone.

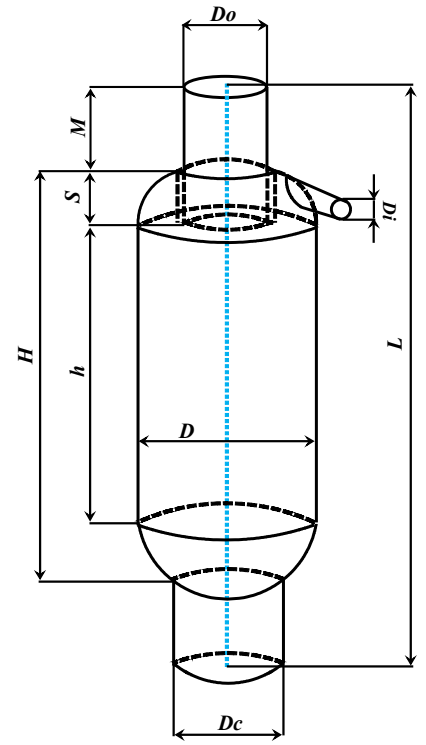

Figure 1 Schematic diagram of ECP

\subsection{Grid generation}

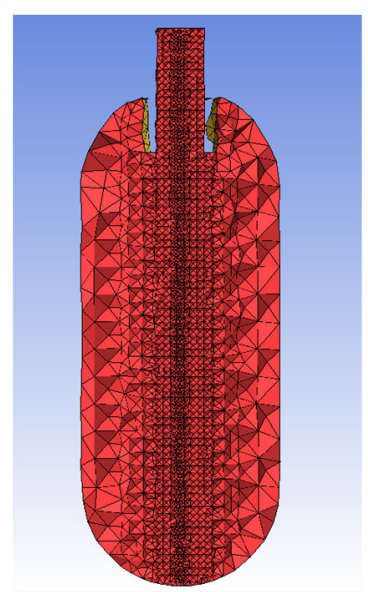

Figure 2 Grid division of the spherical cylindrical ECP

In the whole numerical simulation work, we first establish the solid model of the spherical cylindrical ECP by
SolidWorks, save it as a standard format, import it into ANSYS to extract the fluid domain, and then use ICEM for meshing. When dividing the grid, a hexahedral grid is used [25]. In view of the relatively large potential gradient near the corona line, in order to ensure the calculation accuracy meets the requirements, the grid is encrypted near the central corona line and the flue gas inlet. Figure 2 shows the solid section view.

\subsection{Boundary conditions}

After the mesh is divided, import the mesh file into Fluent and set the boundary conditions, and finally complete the calculation. The boundary conditions of the solution domain in the spherical cylindrical ECP include the flue gas inlet boundary, the flue gas outlet boundary, the ash discharge port, the dust collecting plate, the surface of the corona wire, the exhaust port and other walls of the dust collector. The flue gas inlet is set as the velocity inlet boundary condition, and the flue gas outlet is set as the pressure outlet one. See Table 1 for detailed settings, where $T_{i}$ is the inlet turbulence intensity and $D_{\mathrm{s}}$ is the inlet equivalent diameter.

\subsection{Reliability verification}

The same working conditions in the literature [24] was applied to simulate the dust-removal performance of the spherical cylindrical ECP without electric field, and the comparison curves of dust-removal efficiency were plot in Figure 3. It is not difficult to see that, compared with the simulated result in the literature, the trend of the calculated curve in this paper is not only closer to the experimental data, but also reaches the maximum dust removal efficiency at the same flue gas velocity, and the average relative errors of the two to the experimental data are $4.17 \%$ and $2.95 \%$ respectively, showing that the spherical cylindrical ECP numerical model with magnetic confinement in this study can accurately simulate the particle dust-removal performance.

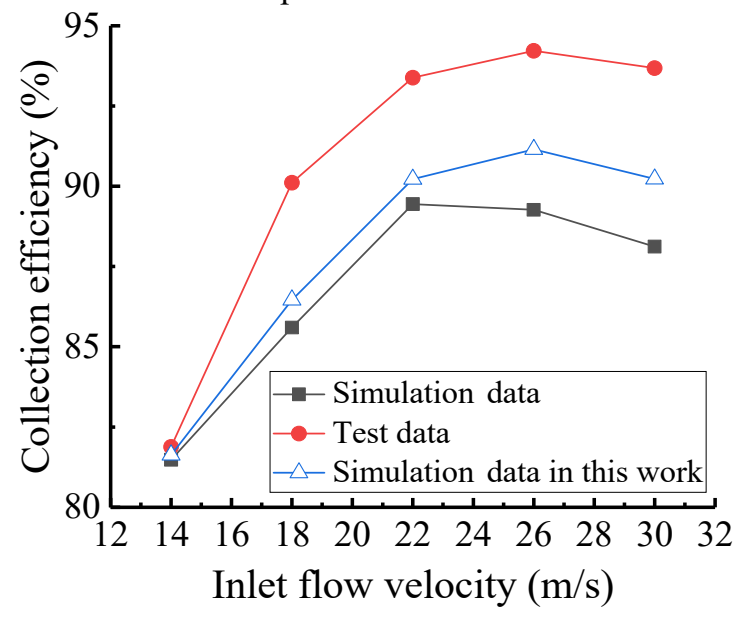

Figure 3 Comparison of collection efficiency 


\subsection{Mesh sensitivity analysis}

In numerical calculations, the number and the quality of grid division will have a great impact on the calculation accuracy. In order to avoid calculation errors caused by improper grid number division, one can select the gas velocities of $20 \mathrm{~m} / \mathrm{s}$ and $32 \mathrm{~m} / \mathrm{s}$, the working voltages of $30 \mathrm{kV}$ and $90 \mathrm{kV}$, and the magnetic induction intensities of $0.0 \mathrm{~T}$ and $1.0 \mathrm{~T}$ for verification. Gradually increase the grid density according to a certain proportion, and the four grid numbers of 161134, 195456, 232006 and 277247 were used.
The overall efficiency and the relative error of the spherical cylindrical ECP with magnetic confinement under the four grid numbers are listed in Table 2. It can be seen that the error of overall efficiency gradually decreases with the increase of the number of grids under any working condition. When the number of grids reaches 277,247 , the errors under all working conditions are the smallest, and they are all less than $1 \%$, which fully meets the requirement of engineering calculation. Therefore, this number of grids was employed in this study. It should be noted that the grid verification covers all calculation conditions and the verification results are credible.

Table 1 Boundary conditions of spherical cylindrical ECP

\begin{tabular}{cccccc}
\hline & Gas Phase & $U$ & $k$ & $\varepsilon$ & Particle Phase \\
\hline v-in & $u=u_{0}$ & $\nabla U=0$ & $3 T_{i}^{2} u_{i}^{2} / 2$ & $0.05 k^{1.5} D_{\mathrm{s}}$ & Escape \\
p-out & Pressure & $\nabla U=0$ & $\partial k / \partial x=0$ & $\partial \varepsilon / \partial x=0$ & Escape \\
Corona wire & No slip & $U=U_{0}$ & Wall-function & Wall-function & Reflect \\
Down-out & No slip & $\nabla U=0$ & Wall-function & Wall-function & Trap \\
Collection plate & No slip & $U=0$ & Wall-function & Wall-function & Trap \\
Other-surface & No slip & $\nabla U=0$ & Wall-function & Wall-function & Reflect \\
\hline
\end{tabular}

Table 2 Grid convergence verification of the spherical cylindrical ECP

\begin{tabular}{|c|c|c|c|c|c|c|}
\hline \multirow{2}{*}{$\begin{array}{l}\text { Voltage } \\
(\mathrm{kV})\end{array}$} & \multirow{2}{*}{$\begin{array}{l}\text { Magnetic field } \\
(\mathrm{T})\end{array}$} & & \multicolumn{4}{|c|}{ Grid Numbers } \\
\hline & & & 161134 & 195456 & 232006 & 277247 \\
\hline \multirow{2}{*}{30} & \multirow{2}{*}{0} & Overall efficiency (\%) & 57.87 & 58.55 & 59.19 & 59.78 \\
\hline & & Relative error (\%) & - & 1.17 & 1.09 & 0.99 \\
\hline \multirow{2}{*}{30} & \multirow{2}{*}{1} & Overall efficiency (\%) & 94.88 & 95.87 & 96.7 & 97.42 \\
\hline & & Relative error (\%) & - & $1.04 \%$ & 0.87 & 0.75 \\
\hline \multirow{2}{*}{90} & \multirow{2}{*}{0} & Overall efficiency (\%) & 89.93 & 91.03 & 91.85 & 92.54 \\
\hline & & Relative error (\%) & - & $1.22 \%$ & 0.94 & 0.75 \\
\hline \multirow{2}{*}{90} & \multirow{2}{*}{1} & Overall efficiency (\%) & 96.3 & 97.21 & 97.91 & 98.33 \\
\hline & & Relative error (\%) & - & 0.95 & 0.72 & 0.43 \\
\hline
\end{tabular}

\section{Results and discussion}

\subsection{Working voltage effect}

In order to illustrate the effect of the working voltage on the overall efficiency of fine particles with R-R distribution in the spherical cylindrical ECP, Figure 4 depicts the change of overall efficiency with working voltage under different gas velocities. The results reflect that:

1) No matter what the gas velocity is, as the working voltage augments, the overall efficiency increases continuously, and the increase reduces gradually, implying that strengthening electrostatic field can improve the collection performance of fine particles in the spherical cylindrical ECP, and the improvement effect at low working voltage is better.

2) Under the same working voltage, with the ascend of the gas velocity, the overall efficiency presents a trend of first increasing and then decreasing, showing that there exists a value of flue gas velocity in the spherical cylindrical ECP, which can make the dust-removal effect reach the best.

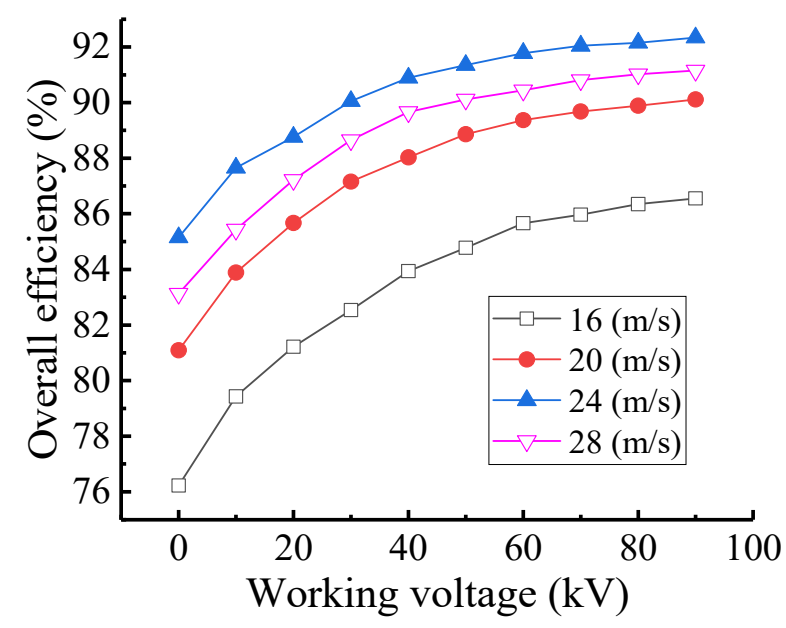

Figure 4 Overall efficiency varying with working voltage 


\subsection{Magnetic confinement effect}

In a magnetic field environment, the working voltage is important operating parameter that affect the trajectory of the particles in the magnetic confinement ECP. The following is to analyze the change rule of overall efficiency of fine particles under different magnetic confinement effects.

The variation curves of overall efficiency with magnetic induction intensity at the inlet velocity of $20 \mathrm{~m} / \mathrm{s}$ are plotted in Figure 5. It can be seen that:

1) Whether the magnetic confinement is applied or not, with the increase of working voltage, the overall efficiency of fine particles increases constantly, and the increase range decreases gradually, proving that the removal performance of spherical cylindrical ECP is improved more significantly by low working voltage.

2) The introduction of magnetic field can significantly improve the overall efficiency of fine particles, and the lifting effect of magnetic confinement gradually weakens as the working voltage increases, implying that the magnetic confinement effect can greatly improve the trapping performance of spherical cylindrical ECP, and becomes more obvious at low working voltage.

3) Under the same working voltage, as the magnetic induction intensity increases, the removal efficiency of fine particles continues to increase, but the increased degree is gradually reduced, which indicates that the magnetic confinement effect in the spherical cylindrical ECP is more obvious at low magnetic induction intensity, leading to the gradual increase of the revenue from the optimization of capture performance.

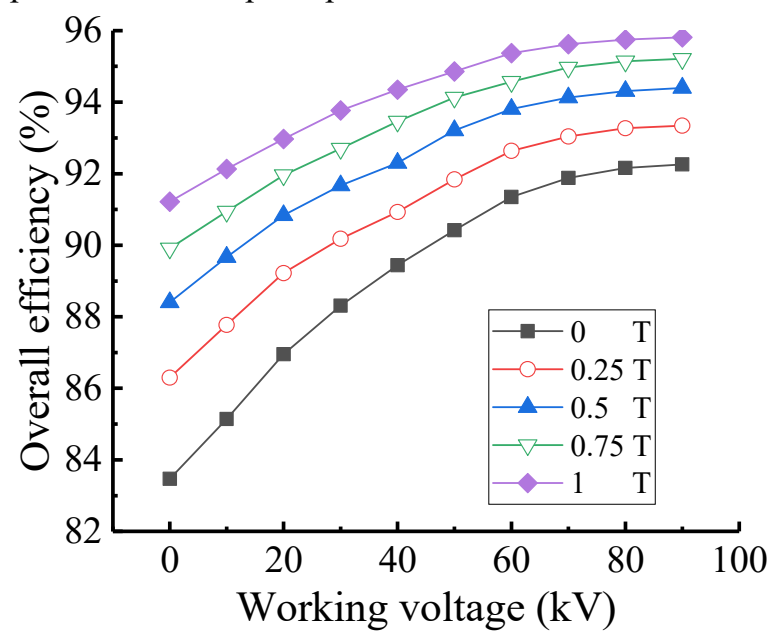

Figure 5 Overall efficiency varying with magnetic field

\section{Conclusions}

In this work, the influences of different operating parameters on the dust-removal efficiency of fine particles with or without magnetic confinement are discussed. The following conclusions are drawn:

1) Under the same gas velocity, increasing working voltage can obviously improve the trapping performance of the spherical cylindrical ECP, and the improvement effect is better at low working voltage.

2) The magnetic field greatly improves the trapping performance of the spherical cylindrical ECP, and the stronger the magnetic confinement effect is, the more significant the improvement effect is. However, the increase of magnetic induction intensity weakens the contribution of magnetic field to the dust-removal efficiency of fine particles.

\section{Acknowledgments}

This work is supported by National Natural Science Foundation of China (11572187); Foundation of Science and Technology Commission of Shanghai Municipality (18DZ1202105, 18DZ1202302).

\section{References}

1. A. Swierczok, M. Jedrusik, D. Luszkiewicz. ELSEVIER BV 104: 103421(2020)

2. J. Grigonyt-Lopez Rodriguez, H. Suhonen, A. Laitinen, J. Tissari, M. Kortelainen, P Tiitta, ALhde, J Keskinen, J Jokiniemi, O Sippula. ELSEVIER LTD 145, 521-529(2020)

3. C. Preger, N. C. Overgaard, M. E. Messing, M. H. Magnusson. Taylor \& Francis 54, 718-728(2020).

4. J.D. Smith, C. Ruehl, M. Burnitzki, W. Sobieralski, H. Dwyer. SCI TOTAL ENVIRON 680, 132139(2019)

5. N. Shahgholian, G. Rajabzadeh. ELSEVIER BV 54, 101323(2019)

6. H. Wu, Q. C. Huan, C. Z. Yang, L. Dong. China Environmental Protection Industry 01, 40-43(2020).

7. J. R. Wang, S. M. Wang, X. D. Duan, J. Wen, J. Y. Tu. POWDER TECHNOL 381, 313-323(2021)

8. A. Izadi, E. Kashani, A. Mohebbi. POWDER TECHNOL 382, 70-84(2021)

9. F. G. Shahna, A. Bahrami, F. Farasati. IND HEALTH 50, 450-457(2012)

10. S. Srinives, T. Charinpanitkul, Tanthapanichakoon W. J IND ENG CHEM 16, 224-229(2010)

11. S. Hayashi, M. Watanabe, Y. Iwase, K. Kanno, K. Proceedings of the 5th Joint Amse/Jsme Fluids Engineering Summer Conference Vol 1, 19251932(2007)

12. P. Baltrenas, A. Chlebnikovas. POWDER TECHNOL 333, 327-338(2018)

13. W. Bo, S. Q. Dong, S. Jie. Xin, R. B. Jin, R. Z. Zhang. IND ENG CHEM RES 57, 11815-11825(2018)

14. H. Ali, F. Plaza, A. Mann. AICHE J 64, 10011012(2018)

15. Y. A. Frolov, K. Nafde, M. F. Vitushchenko. METALLURGIST+, 60, 1228-1238(2017)

16. M. Khorasanimohammadrezaei, N. Aminizadeh. PROCEDIA ENGINEERING 157, 414-421(2016).

17. C. Cortes, A. Gil. PROG ENERG COMBUST 33, 409-452(2007)

18. J. C. Bai, S. Y. Wu, A. S. Lee, C. Y. Chu. J HAZARD 
MATER 142, 324-331(2007)

19. J. Luo, Z. Hao, Y. Dong, J. Zhang, H. Tang. Springer Berlin Heidelberg 262, 695-703(2014)

20. Y. H. Zhang, Y. Z. Liu, H. Huo, D. Chen. Journal of North University of China(Natural Science Edition) 04, 44-48(1998)

21. S. Shaaban. CHEM ENG RES DES 89, 29-38(2011)

22. S. Huang, H. Park, Y.K. Park, Y.M. Jo. AEROSOL SCI TECH 49, 633-642(2015)

23. J. P. Zhang, Z. T. Zha, S. Wang, F. Q. Li, J. Liu, W. G. Pan. J ENG THERMOPHYS-RUS 40, 19361941(2019)

24. Z. G. Yang. Shandong University of Science and Technology ,(2018)

25. S. E. Rafiee, M. M. Sadeghiazad. SEP SCI TECHNOL 52, 903-929(2017) 